Vol. 8 N. ${ }^{\circ} 1$

Enero - Junio del 2021

pp. $161-171$

DOI:

Sugerencia de citación: Gómez-

Ramírez, L. (2021). Las patentes y la pandemia, entrevista con Arjun Jayadev. tiempo\&economía, 8(1), 161-171. https://doi.org/10.21789/24222704.1673

\title{
Las patentes y la pandemia, entrevista con Arjun Jayadev
}

\author{
Patents vs. the Pandemic. Interview with \\ Arjun Jayadev
}

Leopoldo Gómez-Ramírez,

Profesor de Economía, Universidad del Norte, Colombia

leopoldog@uninorte.edu.co

https://doi.org/10.21789/24222704.1673

En coautoría con Joseph Stiglitz, premio nobel de economía, y Achal Prabalha, coordinador del proyecto Access IBSA, el economista hindú Arjun Jayadev' publicó la pieza "Patents vs. the Pandemic". Con ocasión de ella, conversé con él en 400 Voces, programa de entrevistas emitido por la Emisora Uninorte. Lo que sigue son partes de esa conversación.

\section{Leopoldo Gómez-Ramírez (LGR):}

Estimado Arjun, con relación a la pieza "Las patentes vs. la pandemia", la cual escribió con Joseph Stiglitz y Achal Prabalha, y fue publicada en Project Syndicate el 23 de abril del 2020, lo primero que quiero preguntar tiene que ver con el Sistema Mundial de Vigilancia y Respuesta a la Influenza de la Organización Mundial de la Salud. ¿Por qué dicen ustedes que este sistema

\footnotetext{
Arjun Jayadev hizo su doctorado en economía en la Universidad de Massachusetts en Amherst (EE. UU.). Actualmente (julio 2020) es profesor en Azim Premji University (India) y economista senior del Institute for New Economic Thinking (EE. UU.). Sus artículos de investigación académica han sido publicados, entre otras, en las revistas Development and Change, Cambridge Journal of Economics, Journal of Globalization and Development, Journal of Development Economics y American Economic Journal: Macroeconomics.
} 
"epitomiza lo que Amy Kapczynski, de la escuela de leyes de la Universidad de Yale, Ilama ciencia abierta"?

\section{Arjun Jayadev (AJ):}

Cuando la pandemia inició se hizo claro que el verdadero final llegaría cuando tuviéramos una vacuna. Por lo tanto, actualmente hay un gran número de personas de diversas profesiones trabajando en ello. La motivación general detrás de la vacuna para las compañías farmacéuticas es tratar de obtenerla tan pronto como sea posible; lo que obviamente significa que tendrán poder sobre su precio.

Yo intentaba entender cómo era posible que, de hecho, hayamos tenido vacunas contra la influenza por muchos, muchos, años, por décadas, que no son tan caras (al menos no inusualmente caras). Entonces, mientras me encontraba leyendo sobre el Sistema Mundial de Vigilancia y Respuesta a la Influenza en un artículo que escribió Amy Kapczynski, quien hace parte de la escuela de leyes de la Universidad de Yale, descubrí algo de hecho muy asombroso que menciona esta autora. Durante los últimos 30 o 40 años nos hemos vacunado contra la influenza (lo que tanto usted como yo quizás hicimos en Estados Unidos y la gente alrededor del mundo hace), vacuna que fue desarrollada y producida por el Sistema Mundial de Vigilancia y Respuesta a la Influenza, el cual funciona a través de la unión de expertas(os) de todo el mundo que son convocadas(os) una o dos veces al año para analizar y discutir los datos más recientes disponibles sobre las cepas de la influenza y decidir cuáles, en particular, deben incluirse en la vacuna. De forma que hay una enorme red de científicas(os) que abarca 110 países y múltiples laboratorios en distintas naciones, todas(os) reunidas(os) tratando de obtener conjuntamente una vacuna que pueda ser utilizada alrededor del mundo.

Dicha vacuna es financiada por fundaciones, gobiernos y semejantes, por lo que su valor no depende del mercado, razón por la cual es posible establecer precios muy bajos para su comercialización. De forma que esta es una cosa asombrosa, donde la ciencia colaborativa produce algo que se puede usar (o de hecho hemos usado) una, otra, y otra vez, año tras año.

Resulta, entonces, que de hecho podemos encontrar maneras de obtener conjuntamente información farmacéutica muy valiosa, la cual no requiere ni de patentes ni del mecanismo de obtención de ganancias. 


\section{LGR:}

Arjun, estoy de acuerdo cuando ustedes dicen, por ejemplo, que "dado que está únicamente enfocado en proteger vidas humanas en vez de obtener ganancias, [este sistema] tiene una capacidad única para reunir, interpretar y distribuir conocimiento aplicable al desarrollo de vacunas". Sin embargo, obrando un poco de abogado del diablo, para tener algún debate, estoy seguro de que algunos economistas y empresarios dirían que si no existe el incentivo de las ganancias las cosas no van a suceder. Al respecto, ¿qué les diría?

\section{AJ:}

Este es una afirmación interesante. De hecho, es la que a menudo se hace en favor de las patentes. Pero, en realidad, existen dos afirmaciones que comúnmente se hacen en favor de las patentes. Una es que estas son una manera de traer conocimiento al terreno público. La otra establece que se requiere un período de protección tras la generación de nuevo conocimiento, durante el cual es posible obtener ganancias. De otra manera sería simplemente muy costoso, puesto que no habría suficientes incentivos; el primer jugador perdería y no habría motivación. En general, un argumento importante es que el incentivo de las ganancias es el principal impulsor de la innovación.

Ahora bien, suficientemente interesante, se han desarrollado diversos estudios acerca de este tema, donde parece claro que para muchas industrias el incentivo de las ganancias es muy importante. No obstante, también se señala que quizás no es el principal impulsor de la innovación. De hecho, al examinar la historia de la salud pública se evidencian muchos ejemplos en los que las cosas se produjeron sin el incentivo de las ganancias. En realidad, si me preguntan, solo en los últimos 20 o 30 años hemos normalizado este incentivo y los derechos intelectuales que producen ganancias supranormales como elementos necesarios para la innovación.

Quisiera ofrecer un tipo de ejemplo clásico sobre el tema: la vacuna contra la poliomielitis. Esta vacuna, que mejoró la calidad de vida de millones de niñas(os) alrededor del mundo y permitió salvar muchas otras vidas, fue inventada por Jonas Salk, a quien le preguntaron en su momento por qué no patentaba este desarrollo para así obtener ganancias. A lo que él respondió: "Es la vacuna del pueblo, ¿usted patentaría el sol?”. 
Esto todavía me produce lágrimas, a pesar del transcurrir de tantos años. Estamos frente a una innovación que fue extremadamente importante y que no tuvo una motivación económica.

Si analizamos el asunto en un lugar y otro, olvidando a las farmacéuticas y examinando todo el paisaje productivo, encontraremos diferentes lugares en los las que se producen muy buenos productos que no requieren el incentivo de las ganancias. Por ejemplo, en la educación.

Alrededor del mundo observamos, por supuesto, instituciones educativas privadas que obtienen ganancias producto de su operación. Pero también vemos que la educación pública, sin fines de lucro, parece ser la manera en la que podemos crear el mejor sistema educativo.

De manera similar, si analizamos los sistemas de salud alrededor del mundo veremos que los modelos que tienen como finalidad la generación de ganancias son altamente perjudiciales - donde sea que se encuentren-, puesto que estos hacen que la obtención de ganancias esté por encima de las motivaciones humanas que generan algo como un sistema de salud. De forma que estamos viendo muchos sistemas de este tipo, en los que la motivación por ganancias de tipo económico no solo es innecesaria, sino que además se configura en una práctica perjudicial a la luz de los resultados sociales que desearíamos obtener.

\section{LGR:}

Desconozco si existe un equivalente en India, pero en México se usa bastante la frase "juzgan como viven", la cual quiere decir que, a veces, las personas juzgan al otro con base en su propia realidad. En ocasiones considero que detrás de estas ideas, según las cuales las ganancias son el incentivo sin el cual no es posible que las cosas ocurran, lo que en realidad sucede es que quien las arguye piensa que "dado que yo quiero tener ganancias, todas las demás personas también desean lo mismo". ¿Qué opina sobre esto?

\section{AJ:}

Bueno, es un tema interesante. Cuando pensamos en nuestras vidas me pregunto cuántos de nosotros vivimos pensando diariamente bajo el incentivo de las ganancias. Quiero decir, yo sé que los capitanes de las industrias piensan en ello. Sin embargo, a veces pienso en nuestras vidas y en la forma en la que las vivimos, en nuestros hogares, nuestras familias, donde 
obviamente el incentivo de las ganancias no es dominante. En nuestras relaciones más cercanas hay otros motivos, como el altruismo y el cuidado de las/los demás.

Incluso, si gusta, lo mismo sucede en nuestros sistemas productivos. Si les preguntan a los grandes innovadores por la razón detrás de algunas de sus decisiones creo que dirían cosas como "hacer las cosas mejor," "hacer del mundo un lugar mejor", "simplificar ciertos procesos", "lograr ser autosuficientes".

Hay muchos motivos en las economías que no se pueden reducir exclusivamente al incentivo de las ganancias. Esto no significa que dicha variable no sea importante. Es solo que en la vida existen muchas otras motivaciones, que tal vez desde el punto de vista de la Economía usualmente omitimos.

\section{LGR:}

Hablando de Economía como campo de estudio, si le dijera que una gran parte de esta se basa en la creencia de que el incentivo de la ganancia es el impulsor de toda interacción económica, ¿qué me diría?

\section{AJ:}

Diría que es una ficción muy común de los economistas. De hecho, diría que es cierto que el sistema en que vivimos, que de manera general podemos llamar capitalismo, a menudo ha sido dirigido por el incentivo de la ganancia y el interés por la creación de excedentes. Sin embargo, claramente este no es el caso en muchas de nuestras interacciones sociales.

He proporcionado dos ejemplos comunes: educación y salud. De hecho, en el ámbito de la educación y la salud hemos visto al incentivo de las ganancias ir en contra del tipo de cosas que en realidad quisiéramos que pasaran.

En el contexto de la Economía se ha dado, en mi opinión, esta idea que sugiere que si no existe el incentivo de las ganancias las personas tienden a volverse perezosas, se inclinan por no producir, con lo cual se generaría un sistema improductivo en el que la gente viviría en la pobreza, y así por el estilo. Personalmente, no creo que esto sea del todo cierto. También creo que la Economía, como disciplina, está llegando a la realización de que entender el funcionamiento del entorno económico implica que no podemos ignorar las otras motivaciones de los seres humanos. 


\section{LGR:}

Con respecto a lo que acontece en torno a la pandemia, ustedes señalan que últimamente se ha propiciado un nuevo clima de cooperación por parte de la comunidad científica. Comentan también que este hace fácil olvidar que las compañías farmacéuticas han estado durante décadas privatizando los bienes comunales del conocimiento. Por favor, cuéntenos al respecto.

\section{AJ:}

Muchas, muchas, de las innovaciones generadas por las compañías farmacéuticas han sido subsidiadas o creadas por universidades financiadas con recursos públicos. Así que, consideradas apropiadamente, tales innovaciones no deberían ser propiedad privada en lo absoluto. En cambio, estas deberían ser del dominio público. Sobre el tema, Amy Kapczynski, a quien ya mencioné, fue una de las primeras personas en luchar para que las universidades creadoras de este tipo de bienes no buscaran licenciarlos con el objetivo de crear ganancias y privatizar bienes comunales del conocimiento.

En los últimos 25 años hemos visto innovaciones que han recibido algún tipo de respaldo de naturaleza pública y sido objeto del interés de compañías que buscan obtener dominio sobre su propiedad intelectual. Es común ver la innovación de una compañía pequeña absorbida por una firma de gran tamaño.

Las grandes compañías son muy buenas en llevar a cabo experimentos, estudios de mercado y cosas por el estilo. Como resultado, tenemos un sistema muy perverso, en el que las grandes compañías no son necesariamente las más innovadoras. Ellas son, sin lugar a dudas, las que tienen los bolsillos del tamaño suficiente para llevar a cabo experimentación y cosas por el estilo. Esto en sí mismo tiene sus propios problemas, porque cuando ya se han asignado ciertos recursos para llevar a cabo ensayos se cuenta con todo el incentivo de decir que un producto es más de lo que en realidad se trata. Pero bueno, ese es un tema aparte.

El punto central es que mucho del conocimiento detrás de las innovaciones no es generado por una compañía particular, sino que, a menudo, es producido con apoyo público, solo que las farmacéuticas buscan privatizar los bienes comunales para obtener ganancias, en detrimento del bien común. 


\section{LGR:}

Ustedes ofrecen ejemplos específicos relacionados con la COVID-19, señalando que quizás el control monopólico tiene como costo vidas humanas. En particular, mencionan ejemplos relacionados con los kits de pruebas, las máscaras con nivel de seguridad médico y con tres de los más promisorios tratamientos para esta enfermedad, que son remdesivir, favipiravir y liponavir/ritonavir.

\section{AJ:}

De manera temprana apareció la máscara N95, propiedad de la compañía 3M, la cual cuenta aproximadamente cuatrocientas patentes; aunque no todas vigentes. Esto hizo difícil que este producto se manufacturara a gran escala; o por lo menos que se produjera a una escala que no fuera en contra de los derechos de propiedad intelectual. Así que el gobernador de Kentucky, en Estados Unidos, pidió que renunciarán a las patentes para que dicho producto fuera posible, lo cual aconteció al inicio de la pandemia.

Actualmente, la medicina aceptada como tratamiento, es decir, remdesivir, es propiedad de la compañía Gilead, en torno a la cual se ha presentado una situación muy extraña, debido a que el precio de sus acciones ha aumentado varios miles de millones de dólares como resultado de que remdesivir es potencialmente un buen tratamiento. En vista de esta situación, Gilead ha estado de acuerdo con otorgar licencias voluntarias a tres empresas en India, por ejemplo. De esta manera, será más económico producir y vender remdesivir en dicha nación. Por supuesto, esto es algo aún por suceder. No obstante, en países como Brasil, y otros considerados como ingreso medio alto, no existe una obligación expresa para que Gilead o alguna otra compañía suministre estos medicamentos a precios accesibles. De forma que si alguien tuviera que comprar el medicamento en Brasil hoy mismo, este costaría una cantidad enorme, por encima de 4.000 dólares, lo cual resulta ser complicado, a menos que se cuente con algún tipo de seguro que permita acceder al medicamento.

Entonces, estamos frente a un sistema peculiar en el que una compañía tiene el poder de decidir quién puede acceder a determinado medicamento y a qué precio puede hacerlo. En este escenario, a países como Estados Unidos y Reino Unido se les otorga acceso a dicho medicamento a un menor costo, en lo que se configura como un buen ejercicio de relaciones públicas. 
Así mismo, en países pequeños, que no importan grandes volúmenes, están dispuestos a ofrecerlo por una licencia menor. En contraste, en países de ingreso medio y medio alto el acceso a este producto será más complejo.

En síntesis, si continuamos con este tipo de sistema nos veremos ante una situación en la que, por causa de la propiedad intelectual asociada, se generarán diversas clases de resultados extraños alrededor del mundo.

\section{LGR:}

Ustedes hablan de una "elección entre dos futuros". ¿Cuál es el primero?

\section{AJ:}

El primer futuro es el mismo mundo en el que ahora vivimos. Asumamos que no existe este fondo común de propiedad intelectual para combatir la COVID-19 y que simplemente hay que esperar a que aparezcan mejores medicinas. Imagino lo que entonces podría pasar en el futuro.

Básicamente, le estaríamos dando poder sobre la decisión de quién vive o muere a la determinación de precios de las compañías farmacéuticas, esperando simplemente que estas encuentren maneras de obtener ganancias en Occidente, al mismo tiempo que puedan proveer medicinas, o la vacuna, a precios menores para aquellos que no puedan pagarlas. Ante ese escenario tendríamos que trabajar a través del mecanismo de precios, pero en los términos que la compañía productora establezca. Ese es el primer conjunto de resultados.

\section{LGR:}

Precios de monopolio, ¿cierto?

\section{AJ:}

Precios de monopolio, en otras palabras.

Sabemos, a partir de la Economía, que la determinación de precios de los monopolios puede ser efectiva si estos ejercen una discriminación perfecta de precios, lo cual no ocurre en la práctica, por lo que se requiere mucha negociación.

Ahora bien, la segunda opción (el segundo futuro) es que tengamos algo como el Sistema Mundial de Vigilancia y Respuesta a la Influenza, por medio del cual esperamos hallar y mejorar nuestro entendimiento sobre 
esta terrible enfermedad a través la ciencia abierta, en la que toda la información está disponible rápidamente.

En este caso, en primer lugar, tendríamos las ventajas de sumar mucha más información, contar con tamaños de muestra mayores, entender cómo los distintos tratamientos afectan a diferentes poblaciones y aprender de ello mientras al mismo tiempo se están produciendo nuevas medicinas. En segundo lugar, una vez se obtenga una medicina efectiva, esta podrá ser producida al margen de costo, dado que el costo fijo (el costo intelectual) ya fue cubierto por fundaciones, gobiernos y la buena voluntad de las personas. En ese momento será posible producir el medicamento al menor costo posible y maximizar su acceso por parte de la población.

Estos son los dos futuros que puedo vislumbrar como futuros extremos. Aunque también existen situaciones intermedias, estos son los dos futuros extremos que puedo ver. Por supuesto, soy partidario del segundo, ese en el que podamos innovar de una manera mucho más rápida gracias a la ciencia abierta y tener acceso a medicina mucho más económica.

\section{LGR:}

Relacionado quizás con el primer futuro, he leído a personas que afirman que en el momento en que aparezca la vacuna, si llega a suceder, esta va a ser usada por las naciones como herramienta de negociación. ¿Qué piensa de esto?

\section{AJ:}

Se trata de un tema interesante. En este momento una compañía farmacéutica no podría decir que se trata de un asunto que va a considerar. Obviamente, tendría que afirmar que la vacuna será comercializada a un precio muy bajo. Sin embargo, el primer país que desarrolle una vacuna efectiva, si la desarrolla, va a contar con una gran influencia sobre el sistema global. Así que la compañía, bien sea de la Unión Europa, Estados Unidos, Suiza, o incluso de Japón o China, que pueda producir esta vacuna va a obtener un poder enorme sobre el entorno mundial, una situación que considero muy riesgosa. Naturalmente, ningún país querría estar en la posición de suplicarle a otra nación (o compañía) que produzca la medicina que le permita salvar la vida de sus pobladores. 
De forma que este es potencialmente un problema que estaría asociado al primer escenario futuro del que ya hablamos.

\section{LGR:}

Estimado Arjun, permítame hacerle una última pregunta difícil. Quizás soy demasiado pesimista, pero en ocasiones siento que no tenemos ningún tipo de poder. En realidad, ¿considera que tenemos la posibilidad de lograr la segunda opción?

\section{AJ:}

Esta es una pregunta interesante, a la cual quiero responder con historia que deberá hacerlo sentir optimista.

En los años 1999 y 2000 se afirmaba que en África subsahariana se estaba experimentando una crisis del SIDA que acabaría con la vida de más de 25 millones de personas, frente a la cual no había nada que pudiera hacerse. De hecho, algunas personas escribían artículos acerca de cómo, en efecto, dicha situación sería positiva para esta región de África en el mediano plazo, debido a que el estándar de vida de su población aumentaría por efecto de razones maltusianas. Se trataba de una situación verdaderamente horrible.

Al mismo tiempo se estaban desarrollando medicinas que podían ayudar a combatir la enfermedad, es decir, los antivirales. Sin embargo, estos medicamentos eran simplemente muy costosos. Ese era el problema.

A partir de dicha situación, algunos activistas y la sociedad civil alrededor del mundo emplearon mucho tiempo cuestionando al status quo. Entonces, una compañía hindú llamada Cipla empezó a producir la medicina a un precio mucho más barato. En el momento que lo hizo hubo competencia; por cierto, para nosotros las(os) economistas la competencia es un fenómeno positivo ante este tipo de situación, una cosa excelente, porque realmente contribuye a reducir costos: precios al costo marginal. Como resultado de este activismo y toda la presión de la sociedad, así como de las actividades de Cipla, los medicamentos para combatir la enfermedad fueron puestos a disposición de una farmacéutica hindú. Uno o dos años después, las compañías de Occidente comenzaron a disminuir sus precios, al punto que en la actualidad estos medicamentos se encuentran disponibles para millones de pobladores del continente africano. Así, actualmente millones de personas siguen con vida, llevando vidas perfectamente normales, personas VIH-positivas que no hubieran imaginado estar con vida en 1999. 
Por ende, creo que lo que podemos hacer es seguir intentando hablar de soluciones realizables y racionales. Es claro que los sistemas van a continuar presionando, pero creo que es realizable y racional abordar un sistema común y abierto, por la situación que estamos viendo, en vez de un sistema que oprima a las personas. Tenemos evidencia de que tal sistema es posible, razón por la cual escribimos esta pieza (Patents vs. the Pandemics). El Sistema Mundial de Vigilancia y Respuesta a la Influenza es evidencia de que es posible crear muy valiosas piezas de medicina y avances médicos sin la necesidad de privatizarlos y evitar que la gente acceda a ellos.

Por lo anterior, considero que soy más optimista que usted. Sin embargo, también es cierto que esta realidad requiere de mucho esfuerzo y que se desencadenará una enorme reacción, sutil o abierta, por parte de aquellas(os) que prefieren que los negocios sigan como están. 